\title{
Salud Mental en adolescentes de 14 años testigos de violencia intrafamiliar
}

\section{Mental Health in adolescent of 14 years witnesses of familiar violence}

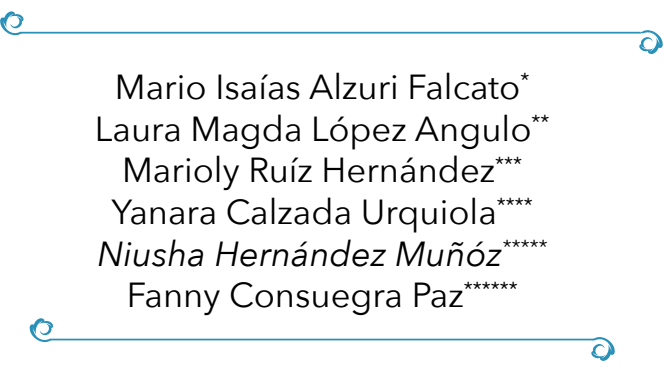

Recibido 18. 06. 2019• Arbitrado 23. 08. $2019 \bullet$

\section{Aprobado 12.09. 2019}

Especialista de 2do Grado en Psiquiatría Infantil. Máster en Psicología de la Salud. Profesor Auxiliar. Universidad de Ciencias Médicas de Cienfuegos. Hospital Pediátrico Universitario Paquito González Cueto. Cienfuegos. Cuba. ORCID: 0000-00018012-3884, marioaf@jagua.cfg.sld.cu

* Licenciada en Psicología. Dra. en Ciencias de la Salud. Profesor Consultante. Universidad de Ciencias Médicas de Cienfuegos. Cuba. ORCID: 0000-0002-8699-5533, psicología6005@ucm.cfg.sld.cu

*** Especialista de 1er Grado en Psiquiatría Infantil. Máster en Atención Integral a la Mujer. Profesor Asistente. Universidad de Ciencias Médicas de Cienfuegos. Hospital Pediátrico Universitario Paquito González Cueto. Cienfuegos. Cuba, ORCID: 00000003-0047-6300, mariolisrh@jagua.cfg.sld.cu

**** Especialista de 1er Grado en Psiquiatría Infantil. Máster en Psicología de la Salud. Profesor Instructor. Universidad de Ciencias Médicas de Cienfuegos. Hospital Pediátrico Universitario Paquito González Cueto. Cienfuegos, Cuba, ORCID: 00000003-2831-3212, dayanara8603@gmail.com

Especialista de 1er Grado en Psiquiatría Infantil. Máster en Atención Integral al Niño. Profesor Instructor. Universidad de Ciencias Médicas de Cienfuegos. Hospital Pediátrico Universitario Paquito González Cueto. Cienfuegos, Cuba, ORCID: 00000002-3771-3149,niusha@nauta.com.cu

Licenciada en Educación Especial. Universidad Pedagógica Conrado Benítez. Máster en Atención Integral a la Mujer. Profesor Asistente. Hospital Pediátrico Universitario Paquito González Cueto. Cienfuegos. Cuba, ORCID: 0000-00017048-3456, fanycp@hosped.cfg.sld.cu

\section{Resumen}

La violencia intrafamiliar provoca secuelas psicológicas en quienes la padecen, sobre todo, cuando ocurre en las primeras etapas del desarrollo, sin embargo, algunos autores plantean que no siempre esto sucede. Objetivo: valorar la salud mental en adolescentes de 14 años testigos de violencia intrafamiliar, pertenecientes a la secundaria básica urbana Frank País García de Cienfuegos, en el período febrero - julio del 2015. Material y Métodos: estudio descriptivo observacional, mixto en el que se aplicó entrevista, pruebas psicométricas y pedagógicas al adolescente, entrevista al familiar y al profesor guía, con triangulación de la información. Resultados: predominaron los adolescentes que convivían con ambos padres casados, en familias extensas, medianas, bigeneracionales y moderadamente funcionales. Prevaleció la violencia intrafamiliar psicológica, cruzada en la pareja y la que dirigía la madre al padre del adolescente. Se identificaron alteraciones emocionales, cognitivas, de aprendizaje y conductuales en los adolescentes de la muestra. Conclusiones: la metodología empleada en esta 
investigación resulta acertada, viable y practicable para valorar la salud mental de los adolescentes testigos de la violencia intrafamiliar.

Palabras clave: maltrato infantil, salud mental, violencia intrafamiliar.

\section{Abstract}

The familiar violence provokes psychological sequelae in who they suffer it, most of all when occurs in the first stages of development, however, some authors propose than no always this it happens. Objective: appraising the mental health in adolescent of 14 years witnesses of familiar violence, refer to Cienfuegos's High School "Frank País García", in the period February - July of 2015. Methods: descriptive observacional, qualitative-quantitative survey in which was applied the interview, psychometrytestings and pedagogical test to the adolescent. As well it was used interview to the relative and to the principal professor, with triangulation of the information. Results: predominated the teens than were living together with both parents, in extensive families, medium, bi-generational and moderately functional. Prevailed chummy violence psychological, crossed in the couple, and the one that the mother was directing to the father. Emotional, cognitive, learning and conductuales alterations were identified in the teens. Conclusions: the methodology used in this investigation proves to be correct, viable and practicable to appraise the mental health of the adolescent witnesses of chummy violence.

Keywords: infantile mistreatment, mental health, chummy violence.

\section{Introducción}

La violencia intrafamiliar se considera como toda acción u omisión cometida en el seno de la familia por uno o varios de sus miembros que de forma permanente ocasione daño físico, psicológico o sexual a otros de sus miembros, que menoscabe su integridad y cause un serio daño a su personalidad y/o a la estabilidad familiar. (Martínez Gómez, 2001).

La violencia es el resultado de la acción recíproca y compleja de factores individuales, relacionales, sociales, culturales y ambientales. Entre los sociales y ambientales se encuentran el no reconocimiento de la violencia como problema de salud, la tolerancia social, las normas, los valores, los mitos, las creencias, los roles, la escasez de recursos económicos. Entre los factores individuales, la edad joven, el abuso del alcohol, la depresión, los trastornos de personalidad. Entre los relacionales, antecedentes de hogares sísmicos, 
el sexismo, los conflictos interpersonales, estresores económicos, disfunción familiar. (Martínez Gómez, 2001)(Clavijo, 2007).

Este fenómeno es considerado como un problema social y de salud, que afecta de manera silenciosa y sistémica a importantes sectores de la población, especialmente a grupos muy vulnerables, contra los cuales amenaza pasar de una violencia endémica a una violencia epidémica en el próximo milenio. (Gateway, 2006).

América Latina es el área con mayor impacto de violencia en el mundo; se reconoce como un problema político y de salud pública que afecta la sociedad y el desarrollo juvenil(Alvarado, 2013).

Particularmente en el tema de protección y atención a los niños, en el año 1989 se firma la Convención de los Derechos de los Niños, a los cuales muchos países se encuentran adheridos, y que pese a los grandes esfuerzos por darle cumplimiento aún se hacen evidentes las dificultades para asegurar los derechos básicos a los integrantes de este sector poblacional (Castellano, 2013).

Martínez Gómez (2001) define la violencia intrafamiliar como toda acción u omisión cometida por algún miembro de la familia, que viole el derecho al pleno desarrollo y bienestar de otro. Se dirige fundamentalmente hacia las mujeres, los niños y los ancianos. Paradójicamente el lugar donde se produce es habitualmente el hogar.

Al revisar los estudios publicados sobre el tema de maltrato infantil, se encuentran con mayor frecuencia, los relacionados con las formas que inciden directamente sobre el niño (maltrato físico, sexual). Muy reducidos los que estudian al menor testigo de la violencia intrafamiliar, sobre todo a nivel de país (Torres Triana, Teseiro Plá y Herrera Perdomo, 2013).

Cuba no está exenta de contar con esta problemática descrita a nivel mundial, aunque existen escasos estudios publicados sobre el tema, se pueden citar algunos que tratan este asunto en cuestión, tal es el caso de la investigación realizada por Arce Gómez DL y col. en el año 2012, donde el 93,7 \% de los menores estudiados recibieron algún tipo de maltrato. Por su parte, Román I y cols. en un estudio realizado en Bayamo plantean que por tramos de edad, el grupo donde la incidencia de agravio es mayor, es el de los menores de 13 años. Dueñas J en su investigación: Violencia intrafamiliar: un análisis desde la psicología humanista publicado en la Revista del Hospital Psiquiátrico de La Habana también aborda esta problemática.(Arce Gómez DL, et al, 2012) (Román I, et al, 2013) (Dueñas J, 2013) 
La violencia es uno de los fenómenos más extendidos en la actualidad y su impacto se advierte no solo en situaciones de abierto conflicto, también en la resolución de problemas, a veces muy simples, de la vida cotidiana. En esa medida, se puede afirmar con certeza, que muchas personas, en alguna etapa de sus vidas, han sido víctimas de violencia. No obstante, ya sea que se trate de mujeres u hombres, la violencia asume características diferentes y sus causas tampoco son las mismasy en la gran mayoría de los casos no es reconocida, como no lo son sus consecuencias físicas y psicológicas para el adulto que resulta víctima directa de ella, ni para el menor que la vivencia como testigo.

Considerando referentes antes mencionados, quedó planteado por los autores de este estudio como problema científico que: no se conoce la repercusión que tiene sobre la salud mental de los adolescentes el ser testigos de la violencia intrafamiliar y en esta investigación se defiende la idea de que los adolescentes testigos de la violencia intrafamiliar, sí presentan afectaciones emocionales, cognitivas, de aprendizaje y/o conductuales.

A partir de lo anterior, el presente estudio se orientó a valorar la salud mental en adolescentes de 14 años testigos de violencia intrafamiliar, pertenecientes a la Secundaria Básica Urbana "Frank País García" de Cienfuegos, en el período febrero - julio del 2015.

\section{Materiales y métodos}

Tipo de estudio: observacional descriptivo. Escenario: Escuela Secundaria Básica Urbana Frank País García de Cienfuegos.

Universo: los 218 alumnos de 14 años que cursaban en el momento del estudio el octavo grado del escenario antes definido. Muestra: los 46estudiantes del universo antes definido que al aplicársele el cuestionario para identificar la existencia de formas de maltrato en su familia de convivencia, tanto la directa (dirigida a su persona) como la indirecta (que dirigía un miembro de la familia a otro), no identificaron la primera y sí, fuera testigos, al menos una vez, de la segunda.

De los 46 estudiantes seleccionados, seis salieron de la investigación por haber sido baja o traslado escolar, lo que representó una pérdida de la muestra de un 13\%, quedaron 40 estudiantes que continuaron en el estudio. La muestra tomada como casos críticos, representó el 18,3\% del universo definido. 
Se seleccionó un familiar de convivencia, en correspondencia con cada adolescente, con liderazgo en la familia, así como capacidad y disposición para responder, lo que constituyó un número de 40.

Las manifestaciones psicopatológicas (síntomas) se consideraron categorías cualitativas, para realizar el análisis de los contenidos se tuvo en cuenta las fuentes siguientes: entrevista médica psiquiátrica, caracterización del adolescente por el profesor, la evaluación psicométrica de los adolescentes, que incluyó la batería de pruebas: (Idare, Idere, Rotter, Escala de Autovaloración). Evaluación Pedagógica. Entrevista Semi estructurada y aplicación de la Prueba de Percepción del Funcionamiento Familiar (FF-SIL) (López Angulo, 2010; Louro Bernal, 2011).

Los adolescentes que completaron el estudio fueron diagnosticados: sin trastorno psiquiátrico o con trastorno psiquiátrico, según la propuesta de los Manuales de clasificación vigentes (Otero OejedaA, 2001).

Se confeccionó una base de datos en Excel y se aplicó triangulación de toda la información teórica y empírica. Los textos se procesaron con Word XP y las tablas se realizaron con Excel XP, se muestran los resultados en tablas de frecuencia y de relación de variables expresados en números y porcientos.

El Proyecto de la Investigación y su informe final, fueron presentados al Consejo Científico del Hospital Pediátrico Universitario Paquito González de Cienfuegos, y aprobados, después de un proceso de discusión y análisis.

Se obtuvo la autorización por escrito del director de la escuela para realizar el estudio, igualmente se les explicó tanto a estudiantes como a sus padres en qué consistiría la investigación y se les tomó su consentimiento informado, no sin antes aclarar que todos los datos obtenidos serían utilizados de forma confidencial y totalmente anónima.

\section{Resultados y discusión}

El 21\% de los adolescentes encuestados de inicio, expresaron haber sido testigos de violencia en sus familias de convivencia. Al comparar este resultado con el de otros países, se pudo observar semejanzas, por ejemplo, España muestra un $10 \%$ de la población infantil, en Canadá se estima entre un 11 y un 23\%, en Estados Unidos algunos estudios sitúan esta cifra entre un 20 y un $40 \%$. A nivel mundial UNICEF estima que la cifra de niñas y niños que sufren esta situación estaría entre los 133 y los 255 millones (Lizana Zamudio, 2014). 
Las características sociodemográficas de la muestra estudiada quedó con la siguiente distribución: El 62,5\% correspondió al sexo femenino y 37,5\% al masculino, lo que se puede explicar por atributos y factores relacionados con el género, lo que hace que las niñas, generalmente, pasen más tiempo en casa, y por otra parte, sean más sensibles de percibir las situaciones de violencia. Hubo predominio de las familias extensas $(47,5 \%)$, de tamaño mediano $(67,5 \%)$ y bigeneracional $(67,5 \%)$.

En el mayor por ciento de los casos (52,5\%), los padres estaban casados, no obstante, llama la atención que el divorcio en los padres alcanzó el 47,5\%. El 50\% de los adolescentes convivía con ambos padres, el $40 \%$ con la madre junto a padrastro u otros familiares y un $10 \%$ con los abuelos. Se infiere que el factor generador de la violencia intrafamiliar, para este grupo, no estuvo determinado, en su totalidad, por el divorcio de los padres y/o los "hogares rotos", que con frecuencia son citados(Martínez Gómez, 2001).

Una parte importante de las familias, 24 para un 60\% calificaron como moderadamente funcional, mientras que solo 12 (30\%), alcanzó la categoría de disfuncional y ninguna la de severamente disfuncional, no percibieron igual la situación de violencia los adolescentes que sus adultos; por otra parte, las más altas puntuaciones recayeron en la Adaptabilidad y Permeabilidad. González Panero expresa al respecto, "una familia que no logra una adecuada adaptación al medio y a los cambios, tiene un mayor riesgo de sufrir perturbaciones destructivas, pudiendo derivar en casos de violencia" (González Panero, 2014, p. 5.).

Estas formas opuestas de percibir la violencia intrafamiliar el adolescente y la familia, muestran una vez más, la necesidad de utilizar diferentes fuentes de información que permitan la triangulación de los datos, para con ello lograr una mayor confiabilidad de los resultados.

Se evidenció un predominio del maltrato psicológico, aunque estuvo presente también el maltrato físico, manifestados por las formas cruzadas o bidireccionales, madre y padre en 12 casos para un 30\%, madre y padrastro en 8 para el 20\%, mientras se encontró que el tercer lugar era ocupado por las madres que agredían a sus esposos en 5 casos para un 12,5\%, obsérvese que en las tres, el adolescente ubica a la figura de la madre en un rol activo en la escena de violencia, en las dos primeras como "coprotagonista" y en la tercera como "victimaria".

Las formas cruzadas de maltrato entre los miembros de la pareja, con predominio de la violencia psicológica, ya fueron reportadas en el informe del Proyecto Carmen de la provincia Cienfuegos en el año 2002 y publicadas en 
el artículo "Prevalencia de la violencia física y psicológica en relaciones de pareja heterosexuales" (López Angulo, Fundora Quintero y Valladarez González, 2015).

Al realizar el análisis de los contenidos de las entrevistas realizadas a los adolescentes estos expresaron una serie de síntomas que fueron agrupados por esferas psíquicas; ocupó el primer lugar la afectividad con 47,5\%, dentro de esta con mayor frecuencia los síntomas de tipo depresivo $(37,5 \%)$, expresados por: tristeza, depresión, decaimiento y ansioso (10\%), referido como alterado, ansioso, nervioso; el segundo lugar fue ocupado por los cognitivos y del aprendizaje (20\%), descritos como preocupado(12,5\%) y desconfiado(7,5\%), que se acompañaron de otras manifestaciones, que fueron aturdido, avergonzado, no aprendo y en tercer lugar los de conducta (10\%) mencionaron intranquilo y agresivo, ambos en 5\%. Se destaca que 9 casos no expresaron síntomas para un $12,5 \%$. (Tabla 1 ).

\section{Tabla 1. Sintomas expresados por el adolescente}

\begin{tabular}{ccclccccc}
\hline Afectividad & No & $\mathbf{\%}$ & Cognitiva & No & $\mathbf{\%}$ & Conducta & No & \% \\
\hline Depresivo & 15 & $\mathbf{3 7 , 5}$ & Preocupado & 5 & 12,5 & Intranquilo & 2 & 5,0 \\
Ansioso & 4 & 10,0 & Desconfiado & 3 & 7,5 & Agresivo & 2 & 5,0 \\
& 19 & 47,5 & & 8 & 20,0 & & 4 & 10,0 \\
$\mathrm{n}=40$ & & & & & & & & \\
\hline
\end{tabular}

Fuente: Entrevista al adolescente

La internalización de los síntomas (tristeza, depresión, ansiedad, preocupación, desconfianza) predominó la externalización (intranquilidad, agresividad), lo que se explica por la mayor cantidad de adolescentes femeninas del estudio, siendo en ellas donde se observa con mayor frecuencia esta forma de expresión de los síntomas, lo que puede ser igualmente explicado por los atributos inherentes al género, en los que ejercen un papel preponderante los factores educativos, psicosociales y culturales.

Estudios que abordan la violencia a partir de las formas de maltrato directo al menor o menor víctima de la violencia de género en la pareja, coinciden en identificar alteraciones emocionales, cognitivas y conductuales en ese orden, predominando los problemas de internalización en las féminas y los de externalización en los varones (Alvarado, 2013; González Panero, 2014).

Por otra parte, artículos que abordan las consecuencias para los hijos testigos de la violencia de género en la pareja, citan, por ejemplo, dificultades en la interacción social, aislamiento, falta de empatía y habilidades 
socioemocionales, problemas de agresividad o inhibición, ansiedad, tristeza, depresión, problemas de aprendizaje y atención y memoria, menor rendimiento académico, hiperactividad, agresividad(Lizana Zamudio, 2014).

En la caracterización del adolescente, ofrecida por su profesor, fue la afectividad el área que con más frecuencia se observó afectada en 18 estudiantes, para un $45 \%$, al describirlo como triste, inseguro, en segundo lugar el área cognitiva y del aprendizaje en 17 adolescentes, que representó el 42,5\%, dadas por distractibilidad, y aprendizaje lento, seguida por el área de la conducta con $27,5 \%$, predominaron la inhibición y/o retraimiento en ocho sobre la hiperactividad y/o agresividad en tres; mientras que en 10 adolescentes no fueron descritas afectaciones, estos representaron el 25\% del total (Tabla 2).

Tabla 2. Caracterización del adolescente por el profesor

\begin{tabular}{ccc}
\hline Áreas afectadas & No & \% \\
\hline Afectividad & 18 & 45,0 \\
Cognitiva & 17 & 42,5 \\
Conducta & 11 & 27,5 \\
Ninguna & 10 & 25,0 \\
\hline $\mathrm{n}=40$ & &
\end{tabular}

Fuente: Entrevista al profesor

Los autores del presente estudio llaman la atención en cuanto a la similitud de frecuencias entre los contenidos que se obtuvieron en la entrevista médico-psiquiátrica y la caracterización del profesor, no solo al coincidir en identificar las categorías afectivo- cognitiva y de aprendizaje en ese orden sino en las unidades expresadas. Lo anterior puede sugerir, que la situación de violencia intrafamiliar en que han estado viviendo los adolescentes, con sus efectos, se convierte en esas presiones afectivas o alteraciones emocionales que provocan el no poder asimilar correctamente la enseñanza escolar, condiciones que rápida y fácilmente generan modificaciones estables de su personalidad que los imposibilitan más y más para la vida escolar normal.

En la Tabla 3 se muestran los resultados de la evaluación pedagógica, en la que 23 adolescentes alcanzaron una calificación que correspondió con el grado cursado, representando el $57 \%$. Obsérvese que en el $43 \%$ restante se diagnosticó un retraso escolar, coincidiendo casi exactamente con el 42,5\% de afectación en el aprendizaje reportado por el profesor en la caracterización del alumno. 
Tabla 3. Grado escolar alcanzado en evaluación pedagógica

\begin{tabular}{ccc}
\hline Grado & No & \% \\
\hline Séptimo & 23 & $\mathbf{5 7 , 0}$ \\
Sexto & 16 & 40,0 \\
Inferior a sexto & 1 & 3,0 \\
Total & 40 & 100 \\
\hline
\end{tabular}

Fuente: Evaluación pedagógica

En este aspecto, aunque las dificultades del aprendizaje tienen un origen multifuncional, se puede considerar que la cifra elevada de adolescentes que no alcanzó el grado escolar que les correspondía, guardó relación con la situación de violencia intrafamiliar en que vivían. Resultados similares son reportados por los estudios de maltrato directo al menor y de menores víctimas de violencia de género, en los que las dificultades de aprendizaje aparecen entre las consecuencias (Alvarado, 2013; González Panero, 2014). Por su lado, Lizana Zamudio (2014), no encuentra dificultades importantes en el área del rendimiento escolar en estas situaciones.

En los test psicológicos aplicados para evaluar los estados emocionales ansiedad y depresión de los adolescentes, se alcanzaron niveles de medio a alto en las cuatro pruebas. Para la ansiedad como estado el $40 \%$ de los adolescentes presentó un nivel medio, mientras que para la ansiedad como rasgo se igualaron los niveles medio y alto en un $45 \%$. Por otra parte la depresión como estado alcanzó niveles de medio a alto en el 37,5\% de los casos y se elevó a 42,5\% para la depresión como rasgo (Tabla 4).

Tabla 4. Estados emocionales según test psicológicos

\begin{tabular}{ccccccccc}
\hline Nivel & $\begin{array}{c}\text { Ansiedad estado } \\
\text { No \% }\end{array}$ & \multicolumn{2}{c}{$\begin{array}{c}\text { Ansiedad rasgo } \\
\text { No \% }\end{array}$} & \multicolumn{2}{c}{$\begin{array}{c}\text { Depresión estado } \\
\text { No \% }\end{array}$} & \multicolumn{2}{c}{$\begin{array}{c}\text { Depresión rasgo } \\
\text { No \% }\end{array}$} \\
\hline Alto & 11 & 27,5 & 18 & $\mathbf{4 5 , 0}$ & 15 & $\mathbf{3 7 , 5}$ & 17 & $\mathbf{4 2 , 5}$ \\
Medio & 16 & $\mathbf{4 0 , 0}$ & 18 & $\mathbf{4 5 , 0}$ & 15 & $\mathbf{3 7 , 5}$ & 13 & 32,5 \\
Bajo & 13 & 32,5 & 4 & 10 & 10 & 25,0 & 10 & 25,0 \\
Total & 40 & 100 & 40 & 100 & 40 & 100 & 40 & 100 \\
\hline
\end{tabular}

Fuente: Propia

Al completar la evaluación de los 40 adolescentes, por medio de las fuentes directas e indirectas de información, se encontró que entre 45 y $50 \%$ presentaron alteraciones, sobre todo, en las esferas emocional - cognitiva y de la 
conducta, que, por las dificultades que generaban para la actividad creadora, complejidad y estructuración, y el malestar que provocaban, cumplieron las pautas de trastorno mental.

Lizana Zamudio (2014) revela que el 73,3\% de los niños y niñas de su estudio, presentaron sintomatología suficiente para ser considerados como algún tipo de trastorno psicopatológico según DSM-IV, mientras que el 26,7\% que no presentan trastornos, tienen sintomatología subclínica, sobre todo síntomas depresivos y ansiosos.

Al realizar la triangulación de toda la información teórica y empírica, se observó coincidencias interesantes:

El primer lugar ocupado por las alteraciones emocionales: identificadas en la entrevista médica $(47,5 \%)$, caracterización del profesor $(45,0 \%)$ y test psicológicos (ansiedad como rasgo 45,0\% y depresión como rasgo 42,5\%), se evidenció la similitud de frecuencia en la entrevista médica, la caracterización del profesor y los test psicológicos.

Hubo predominio de las manifestaciones depresivas: identificadas en la entrevista médica $(37,5 \%)$, caracterización del profesor $(45,0 \%)$ y test psicológico (depresión como rasgo 42,5\%), igualmente se mostró la similitud de frecuencias que resultaron de la entrevista médica, la caracterización del profesor y el test psicológico, sobre todo en los dos últimos.

El segundo lugar ocupado por las alteraciones cognitivas y de aprendizaje: identificadas en la entrevista médica $(20,0 \%)$, caracterización del profesor $(42,5 \%)$ y evaluación pedagógica $(43,0 \%)$, hubo coincidencia casi exacta entre la caracterización del profesor y la evaluación pedagógica.

Como principal limitación del trabajo, se reconoce, el pequeño número de casos estudiados, lo que no permite la generalización de los resultados, es por eso que los autores pretenden con estos, mostrar, más que demostrar.

\section{Conclusiones}

Este estudio ha contribuido al conocimiento de las características sociodemográficas y de la violencia intrafamiliar en adolescentes testigos de la violencia.

Se demuestra que la metodología empleada en esta investigación fue acertada, viable y practicable para valorar la salud mental de los adolescentes testigos de la violencia intrafamiliar basada en conceptos aplicables a nuestra realidad. 
La combinación de fuentes y de procedimientos facilitó integrar las cualidades de la psicodinámica familiar con las alteraciones en las esferas afectivas, cognitivas y conductuales de los adolescentes de la muestra.

Se comprobó en la muestra de este estudio que los adolescentes testigos de la violencia intrafamiliar presentan trastornos mentales.

\section{Referencias}

Alvarado, A. (2013). La violencia juvenil en América Latina. Estudios Sociológicos, XXXI (91), 229-258. https://www.redalyc.org/pdf/598/59830136009.pdf

Arce Gómez, D., Castellanos, M., Reina, C. (2012). Algunos factores psicosociales del maltrato infantil en escolares de la enseñanza primaria. Medisan, 16(12):1891, Disponible en http://scielo.sld.cu/pdf/san/v16n12/san111612.pdf

Castellano, B. G. (2013). Por qué la medicina de la adolescencia. Temas escogidos. Adoleca, 1-6. Disponible https://www.adolescenciasema.org/por-que-medicinade-la-adolescencia-g-castellano-barca-revista-adolescere-2013-i-2-07-14/

Clavijo, A. (2007). Crisis y Salud Familiar. En: Crisis, familia y psicoterapia. 2da Edición, Camagüey, Cuba.

Otero Oejeda, A. (2001). Tercer Glosario Cubano de Psiquiatría. La Habana: Hspital Psiquiátrico de La Habana.

Child Welfare Information Gateway. (2006). Consecuencias a largo plazo del maltrato de menores [Internet]. Disponible en: http://www.childwelfare.gov/pubs/ factsheets/long_term_consequences.cfm.

Dueñas, J. (2013). Violencia intrafamiliar: un análisis desde la psicología humanista. Rev Hosp Psiquiátr La Habana, 10(1), 1-6, Disponible en http://www.medigraphic. com/pdfs/revhospsihab/hph-2013/hph131p.pdf.

González Panero, L. (2014). Violencia familiar. El niño como víctima. Grado en maestro de educación infantil, universidad de Cantabria, Disponible en https:// repositorio.unican.es/xmlui/handle/10902/5450.

Lizana Zamudio, R. (2014). Problemas psicológicos en niños y niñas víctimas de la violencia de género en la pareja. Tesis Doctoral. Universidad Autónoma de Barcelona. Disponible ne https://www.tdx.cat/bitstream/handle/10803/285393/rlz1de1. pdf? sequence $=1 \&$ isAllowed $=y$

López Angulo, L. (2010). Compendio de Intrumentos de Evaluación Psicológica. La Habana: Editorial Ciencias Médicas. 
López Angulo, L., Fundora Quintero, Y., Valladarez Gonzpalez, A. (2015). Prevalencia de la violencia física y psicológica en relaciones de pareja heterosexuales. Finlay, 5(3), 10-18.http://scielo.sld.cu/scielo.php?script=sci arttext\&pid $=$ S2221-24342015000300003

Louro Bernal, I. (2011). Manual de Evaluación y Orientación para la Salud Familiar. La Habana: Grupo Asesor Metodológico en estudios de salud de la familia.

Martínez Gómez, C. (2001). Factores de riesgo. Salud Familiar. La Habana: Editorial Cientifico Técnica.

Román I, Román M, Zayas M. (2013). Violencia intrafamiliar en adolescentes. Policlínico René Vallejo Ortiz, Bayamo, Disponible en http://www.codajic.org/ sites/www.codajic.org/files/75\%20-\%20Violencia\%20intrafamiliar\%20\%20 en\%20\%20adolescentes.pdf.

Torres Triana, A., Teseiro Plá, M., Herrera Perdomo, D. (2013). Caracterización del maltrato psicológico infantil en el área de salud del Policlinico "Reynold García". Revista Médica Electrónica, 35 (6), 596-605, http://scielo.sld.cu/pdf/rme/v35n6/ tema04.pdf. 